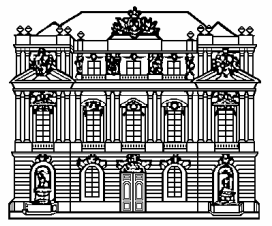

des theoretisch wissenschaftlichen Teiles

\title{
8 Resümee und Empfehlung
}

\section{Projektwerber und Projektleitung:}

INSTITUT FÜR STRASSEN- UND VERKEHRSWESEN (ISV)

Technische Universität Graz

Rechbauerstraße 12/II, A-8010 Graz

Projektleiter: Univ.-Prof. Dr.techn. Werner GOBIET

Projektpartner:

INSTITUT FÜR ZIVILRECHT (IZR)

Universität Innsbruck

HERRY CONSULT GMBH

Em. Univ.-Prof. Dr. Peter FALLER

\section{VERFASSER: FORSCHUNGSGRUPPE Alp-EmiV}

Univ.-Prof.Dr.techn. Werner GOBIET (ISV, Projektleiter)

Em.Univ.-Prof.Dr. Peter FALLER

Dr.techn. Markus FREWEIN (ISV, Projektmanagement)

Ass.-Prof. Mag. Dr. Peter JORDAN (IZR)

Dr. Max HERRY (Herry Consult Gmbh)

unter Mitarbeit von

Martin VILHAR (ISV)

ISBN-10 3-7001-3778-8

ISBN-13 978-3-7001-3778-8

doi: 10.1553/alp-emiv

http://epub.oeaw.ac.at/alp-emiv

Graz, im Juni 2006 


\section{Resümee und Empfehlung}

\subsection{Resümee}

Im Rahmen der Untersuchungen durch die Mitglieder des Forschungsprojektes Alp-EmiV wurden folgende Ziele erreicht:

- Die Größe des Problemfeldes wurde ermittelt.

- Der insgesamt vorhandene Forschungsbedarf wurde aufgezeigt, und zwar:

- Einbringung des Anlagenrechts als grundsätzlicher Baustein zur Lösung regionaler verkehrlicher Immissionsprobleme

- Ansätze zur Verkehrssteuerung in ökologisch sensiblen Gebieten

- Emissionsrechtehandel (Cap and Trade) als marktorientierter Ansatz zur ökologischen Verkehrssteuerung

\subsection{Empfehlungen und Handlungsbedarf}

Was auch immer für Maßnahmen bzw. Maßnahmenbündel für eine ökologische Verkehrssteuerung gesetzt werden, ein EU-Rechts konformer Steuerungsmechanismus ist notwendig. $\mathrm{Zu}$ berücksichtigen wären bei Einführung jedweder Maßnahmen auf jeden Fall folgende Punkte:

- die Diskriminierungsregel (als Prinzip),

- das Wettbewerbsrecht darf nicht ausgehöhlt werden,

- der Marktzutritt darf nicht versperrt werden,

- Differenzierung der Fahrzeuge ist vorzunehmen,

- Zuschuss und Beihilferegelungen der EU muss eingehalten werden,

- auf eine interne Bezuschussung wäre Acht zu geben (z.B. dass die Euro 0 Fahrzeuge die Euro 5 Fahrzeuge unterstützen),

- Ausnahmen müssten gerechtfertigt werden (grundsätzlich sind Diskriminierungen sachlich zu rechtfertigen: z.B. Nahversorgung o.ä.)

- Ausweichmöglichkeiten auf die Bahn oder andere Routen müssten zur Verfügung gestellt werden (keine Totalblockade bestimmter Routen).

Ein zentrales Anliegen dieses Untersuchungsauftrages war es, die Frage zu klären, ob der in den Sektoren Energiewirtschaft und Industrie nach langer Vorbereitung Anfang 2005 in den EU-Ländern gestartete Handel mit CO2-Zertifikaten ein brauchbarer Ansatz für eine umweltorientierte und zugleich nach marktwirtschaftlichen Regeln funktionierende Steuerung des Verkehrs sein kann.

Die Antwort auf diese Frage ist ein prinzipielles „Ja“, aber mit deutlichen Hinweisen auf eine Reihe von Unterschieden zwischen System Energie und System Verkehr, die im Vorfeld und im Fall der praktischen Anwendung zu beachten sein werden. Diese Vorfeldprobleme dürfen nicht unterschätzt werden. Die Adaptie- 
rung eines Modells, das für ein globales, d.h. die Erde als Ganzes betreffendes Problem entwickelt wurde, muss zur Anwendung auf den Verkehr in einem sensiblen Teilraum mit großer Behutsamkeit erfolgen. Während es beim weltweiten CO2-Problem gleichgültig ist, an welchem Punkt der Erde die CO2-Reduktion erfolgt, können bei einer regional eingegrenzten Verkehrssteuerung (wie z.B. im Alpenraum) nur jene Emissionsreduzierungen als Positivum gewertet werden, die in der betreffenden Region nachweisbar sind.

Vor allem ist zu beachten, dass es innerhalb des Verkehrssystems mehrere Verantwortungsebenen gibt, die als Zugriffspunkte für eine Emissionsreduzierung gewählt werden können (Infrastruktur im Falle anlagenrechtlicher Behandlung, Verkehrsbetrieb, Fahrzeug, Treibstoff, Verkehrsorganisation).

Weiters wird auf eine Reihe von Fragen zu achten sein, die sich aus der in jüngster Zeit aufgekommenen Kritik am CO2-Zertifikatehandel herleiten lassen (GratisErstausgabe der Zertifikate? - Welche Stückelung? - Erfordernis einer Plattform für den Emissionsrechtehandel - Erfordernis eines Monitoringsystems zur Fernhaltung von systemexternen Störfaktoren und zur Überwachung der Systemeffizienz).

Insbesondere dem letztgenannten Punkt muss bei der nun erforderlichen Modellspezifizierung besondere Beachtung geschenkt werden: Der Aufwand für ein nach marktwirtschaftlichen Regeln funktionierendes umweltorientiertes Verkehrssteuerungssystem für den Alpenraum muss in einem vertretbaren Verhältnis zu den mit dieser Verkehrssteuerungsvariante erreichbaren Effekten stehen.

\subsection{Ausblick Realisierungsstudie (Pilotstudie)}

Das Forschungsprojekt Alp-EmiV

- Die vorliegende Grundlagenuntersuchung liefert allgemein gültige Aussagen zur emissionsgesteuerten Verkehrslenkung, ist aber:

- noch nicht anwendungsorientiert, d.h. noch nicht hinreichend praxisnah,

- noch nicht raumspezifisch und zeitlich eingegrenzt,

- noch nicht mit einem datenbasierten Mengengerüst ausgestattet,

- noch nicht mit den spezifischen Anforderungen der betroffenen Akteure vernetzt,

- noch nicht genügend auf die Umsetzbarkeit unter realen Bedingungen hin überprüft.

\subsubsection{Ziele für eine Fortsetzungsstudie}

Ziele der Fortsetzungsstudie sind demnach: 
- Operationalisierung des Systems Alp-EmiV auf Basis der Erkenntnisse der Grundlagenuntersuchung

- Ausarbeitung und detaillierte Prüfung eines rechtlich gesicherten Rahmens (EU-Rechtskonformität)

- Konkrete Prüfung bestehender Modelle der ökologischen Verkehrslenkung, wie z.B. des Schweizer Modells der Alpentransitbörse (Informationsaustausch Schweiz-Österreich)

- Aufzeigen des Zusammenhangs zwischen der ökologisch begründeten Maut und der aktuellen Entwicklungen der Wegekostenrechnung

- Entwickeln des logistischen Konzeptes der emissionsgesteuerten Verkehrslenkung

- Notwendige dynamische und statische Datenbasis

- Dynamische Preisbildungsverfahren

- Dynamische Berechnung der maximalen ökologisch begründeten Verkehrsbelastung (Cap)

- Konkretisieren des Cap- and Trade-Systems

- Kontrollsystem zur Einhaltung der Grenzwerte 